\title{
COMPARATIVE STUDY ON MICROLEAKAGE OF COMPOSITE INLAYS USING VARIOUS RESIN LUTING CEMENTS
}

WA. Annuar, H. Abdullah. Comparative study on microleakage of composite inlays using various resin luting cements. Annal Dent Univ Malaya 2003; 10: 27-32.

\section{ABSTRACT}

This study is conducted to compare two resin luting cements (Rely XTMARC3M and Compolute ${ }^{\text {TM }}$ ESPE) on their microleakage with one composite inlay system (Filtek ${ }^{\mathrm{TM}} \mathrm{Z} 250$ Universal Restorative Materials, 3M). Thirty conventional inlays, Class II MOIDO cavity with gingival margin $\mathrm{I} \mathrm{mm}$ above the cementoenamel junction, were prepared in premolar teeth. The composite inlays were fabricated directly on the prepared teeth using layering technique. Fifteen of the inlays were cemented with RelyTN ARC and fifteen with Compolute ${ }^{\mathrm{TM}}(\mathrm{ESPE})$. The specimens were kept at $37^{\circ} \mathrm{C}$ for 10 days before thermocycling and immersed in methylene blue $2 \%$ solution for 24 hours. The teeth were sectioned mesio-distally. The site and degree of leakage of each section was scored using a visual scoring system under a stereomicroscope at a magnification of $1.5 \mathrm{X}$. Results showed that there was no significant difference in'leakage extent ( $p>0.05$ ), between Rely $\mathrm{X}^{\mathrm{TM}} \mathrm{ARC}$ and Compolute ${ }^{\mathrm{TM}}$. For both materials, leakage occurred most commonly within the enamel surface and between the cement-tooth interfaces. None of the leakage occurred between inlay - cement interface. There is no significant difference in the extent of leakage between Rely XTMARC compared to Compolute ${ }^{\mathrm{TM}}$.

Key words: composite inlay, microleakage

\section{INTRODUCTION}

Advances in dental composite techniques, combined with the ability to produce an aesthetic restoration that is bonded to tooth structure have lead to increased clinical usage of composite in recent years.

Despite improvements in the wear resistance of some of the new materials, problems remained with the use of direct composites especially in the Class II situation. Poor marginal adaptation and microleakage have been identified as problems at the cervical margins of Class II composites (1). Polymerisation shrinkage of the composite and poor adhesion of the material to cervical tooth structure has been implicated in this regard (2). Whilst the development of visible light cured composites has been of major benefit, the inaccessibility of deep confined proximal areas to the light curing source in the Class II situation creates the

\author{
WA. AnnuarI, H. Abdullah² \\ 1 Graduate Student \\ 2Associate Professor \\ Department of Conservative Dentistry, \\ Faculty of Dentistry, \\ University Malaya, \\ 50603 Kuala Lumpur, \\ Malaysia \\ Corresponding author - Hadijah Abdullah
}

potential for poor marginal adaptation because of inadequate cure of the composite in the region (3).

Manufacturers have responded to these problems by introducing composite inlay systems, which utilized direct and/or indirect techniques. A high degree of post curing of composite inlays are assured before the restorations are bonded to the tooth and also the adverse effects of polymerisation contraction can be minimized (4). Post curing composite seems to be advantageous in terms of occlusal and proximal wear compared with direct applied resin base composite (5).

Currently, there are three main types of luting cements commercially available for composite or ceramic inlays; resin composite cement, polyacidmodified resin composite (compomer), and resinmodified glass-ionomer cement(RMGIC) (6).

According to the method of polymerisation, resin composite cements could be classified into three main categories: self cured cements, used mainly for cementation of metallic restorations and posts; light cured cements, used for cementation of porcelain veneers; and dual cured cements, used for cementation of ceramic inlay and onlay restorations, indirect resin composite restorations and ceramic crowns (7).

The advantage of using dual cure luting agents is to reduce the problems associated with inadequate polymerisation of composite resin in areas distant from the light source (3).

In addition to improvement in c;omposite systems and restoration techniques there has been a steady progress in treatments for bonding resins to tooth tissues. Some recently developed dentine bonding agents make use of conditioning liquids, which render the dentine amenable to bonding at the same time as etching the enamel, thus simplifying the resin bonding of a restoration. These dentine bonding agents are able to infiltrate into the etched dentin and form hybrid layer which may reduce microleakage (8). 


\section{OBJECTIVES OF STUDY}

The objective of this study is to make a comparison on microleakage of two resin luting cements in a composite inlay system. The two resin luting cements were used because they have the same components as the composite inlay system. This will enable chemical bonding to form between the inlay and luting media. Each of the luting cement used a different primer system that was recommended by its manufacturer.

\section{MATERIALS AND METHODS}

Two resin luting cement namely Compolute ${ }^{\mathrm{TM}}$ (3M ESPE) and RelyX ${ }^{\mathrm{TM}}$ ARC (3M) were used with the universal composite FiltekTMZ250 (3M).

\section{Preparation of specimens}

Thirty extracted non-carious premolar teeth were stored in $0.5 \%$ Chloramine at room temperature for one week and then stored in distilled water at temperature of $4^{\circ} \mathrm{C}$. The calculus and soft tissue remnants were removed from the selected teeth using ultrasonic scaler. The teeth were randomly selected and separated into 2 groups of fifteen.

\section{Group A - Cemented with RelyXTMARC (3M)}

Group B - Cemented with Compolute ${ }^{\mathrm{TM}}$

(3M ESPE)

\section{Restorative procedures}

Each tooth was mounted on a plaster box for easier preparation of the cavity. Water-cooled high-speed handpiece with tapered fissure diamond burs (ISO 8036 314) was used to prepare conventional Class II (MO)/(DO) inlay cavities where the gingival floor of the cavity was placed about $1 \mathrm{~mm}$ above cementoenamel junction. All cavities were prepared in the dimension of $2.0 \mathrm{~mm}$ in width and $1.5 \mathrm{~mm}$ in depth (Figure la \& lb).

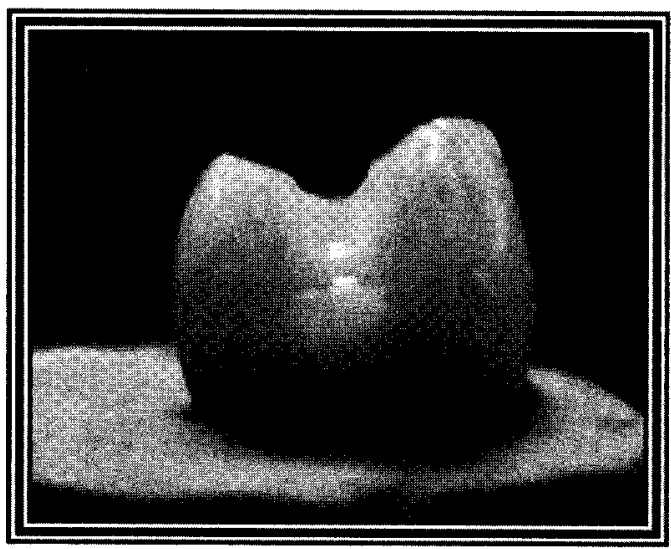

Figure la

\section{Fabrication of composite inlays}

The composite inlay (Filtek Z 250, 3M) was constructed directly on the prepared tooth. A separating agent (Brilliant D.I Coltene Inc., Calf.) was applied to the cavity before the fabrication of these inlays. The composite (Filtek Z 250, 3M) was applied using layering technique whereby the proximal walls were added incrementally along the buccal and lingual surface and light - cured between the applications for 40 seconds to enhance the polymerisation reaction. The preparation border was marked with pencil for easier identification of margins. The cured inlays after being removed from the tooth preparation were trimmed of excess material using white stone burs (Shofu, Dura White Stone, Japan). The marginal adaptation was checked by running a sharp probe along the margins. These inlays were polished with Enhance ${ }^{\circledR}$ polishing paste (Enhance ${ }^{\circledR}$ Composite finishing \& polishing system, Densply) according to the manufacturer's instructions. Prior to cementation, the fitting surfaces of the inlays were sandblasted with 50 Jlm aluminium oxide particles using Basic Professional Sandblaster (Renfert, D78247, Hilzingen, Germany).

\section{Cementation of composite inlays}

Fifteen of the inlays were cemented with resin luting cement RelyTMX ARC, (3M) and fifteen with Compolute $^{\mathrm{TM}}$ (ESPE). Manipulation of the cement was carried out at room temperature following the manufacturer's instructions.

\section{The test group}

\section{Group A - Cemented with Rely тм X ARC (3M)}

For Rely-X ARC (3M) group, the cavities were etched with 3MTMScothbondTMEtchant for 15 seconds. These were then rinsed thoroughly under running water for 10 seconds and dried with damp cotton pellet. Then 3MTMSingle Bond Dental Adhesive System was applied to etched enamel and dentine and dried for 5 seconds and light cured. Appropriate amount of Rely

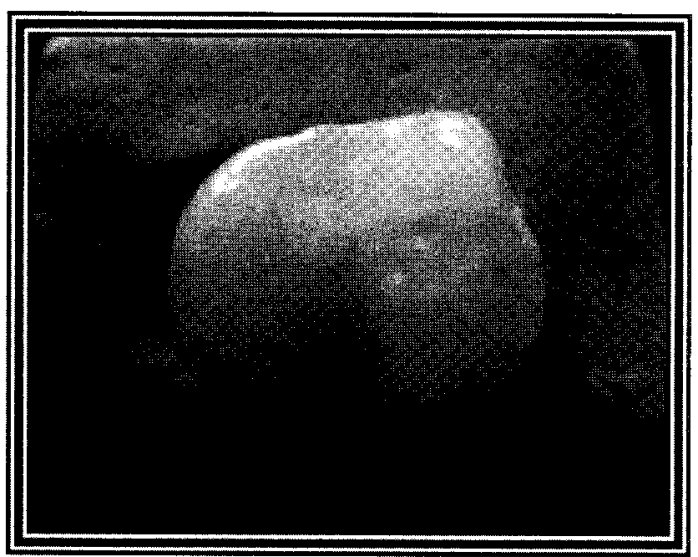

Figure $1 b$

Figure la: The proximal box of the cavity preparation. Figure Ib: occlusal view of the cavity preparation.

Approximate size of the cavity $-2.0 \mathrm{~mm}$ in width and $1.5 \mathrm{~mm}$ in depth. 
- X ARC resin cement was dispensed onto a mixing pad and mixed for 10 seconds. The mixed cement was allplied to the internal surface of the inlays using a small brush. The inlays were held in place by applying finger pressure via a burnisher at the centre of each inlay. Excess cement was removed from all surfaces with a cotton pellet and each surface was cured for 40 seconds using the visible light - cure unit (Coltene®, Coltlux 3). These inlays were than polished with Enhance ${ }^{\circledR}$ polishing kit (Enhance ${ }^{\circledR}$ Composite finishing \& polishing system, Dentsply).

\section{Group B - Cemented with Compolute ${ }^{\mathrm{TM}}($ ESPE)}

For Compolute ${ }^{\mathrm{TM}}$ (ESPE) the enamel and dentine were etched using the total etch technique (EBS® Multi). Etching was carried out for 15 seconds. This was then rinsed thoroughly under running water for 10 seconds and dab dry with cotton pellet. The primer (EBS ${ }^{\circledR}$ Multi Primer) and the bonding agent (EBS® Multi Bonding) were applied to etched enamel and dentine for 20 seconds without light - curing. Appropriate amount of Compolute ${ }^{\mathrm{TM}}$ resin cement was dispensed onto a mixing pad and mixed for 10 seconds. The mixed cement was applied to the internal surface of the inlays using a small brush. The inlays were held in place by applying finger pressure via a burnisher at the centre of each inlay. Excess cement was removed from all surfaces with a cotton pellet and each surface was cured for 40 seconds using the visible light - cure unit (Coltene ${ }^{\circledR}, \quad$ Coltlux 3). Excess cement at the proximal surface was removed. These inlays were then polished with Enhance ${ }^{\circledR}$ polishing kit (Enhance® Composite finishing \& polishing system, Dentsply).

\section{Thermocycling procedure}

\section{Prior to thermocycling}

Ten minutes after cementation the teeth were then stored in distilled water and kept at $37^{\circ} \mathrm{C}$ for 10 days before thermocycling and were then immersed in the dye solution.

\section{Thermocycling regime}

Both groups were thermocycled for 500 times between $15^{\circ} \mathrm{C}$ and $45^{\circ} \mathrm{C}$ with dwell time 15 seconds in each bath (Neslab FTC-350, GP200, USA) and 5 seconds interval between them in the distilled water.

\section{Evaluation of microleakage}

\section{Preparation prior to sectioning}

After thermocycling the specimens were taken out, dab dry with a tissue paper and the root apex sealed with sticky wax. The teeth were coated with a layer of nail polish while $1.0 \mathrm{~mm}$ of the margins of the restoration were kept free of any coating. The coated teeth were then immersed in $2 \%$ methylene blue solvent for 24 hours. After removal from the dye, the coating was removed and the teeth were thoroughly washed in water and dab dry with tissue paper. The

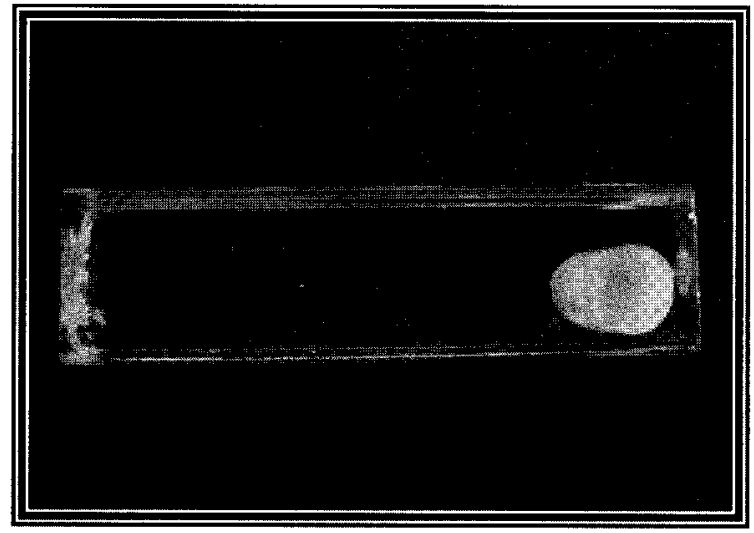

Figure 2: Specimen embedded in epoxy resin (Mirapox 950-230) in the plastic cuvettes (Dispolab Kartell) prior to sectioning.

roots were sectioned $3 \mathrm{~mm}$ below cementoenamel junction by using a straight handpiece with carborandum disc and embedded in the Epoxy resin (Mirapox 950-230) in the plastic Cuvettes (Dispolab Kartell) (Figure 2).

\section{Sectioning of specimens}

The teeth were sectioned longitudinally in the mesio-distal plane at the mid line of the restoration. Sectioning of teeth were carried out by using low speed saw (Buehler, 11-1180 Isometa).

\section{Microleakage evaluation procedure}

Two halves of the specimens were viewed under a light stereomicroscope $15 \mathrm{X}$ magnification (MEIJIZoom Stereo EMZ-1 7613). The half that showed more leakage was chosen for evaluation. All specimens were examined three times for microleakage with a three day period between each evaluation. Each evaluation was carried out independently without reference to previous score. This was to eliminate evaluator's bias.

\section{Criteriafor microleakage evaluation}

The site and degree of leakage of each section was scored using the scoring system as shown in (Figure $3)$.

\section{Data analysis}

The severity of micro leakage data was subjected to statistical analysis by using Chi-Square test.

\section{RESULTS}

The dye penetration was subjectively scored using the scoring system as described in Figure 3.

Chi-Square Test showed that there was no significant difference in microleakage between the two resin cements (Rely X ARC and Compo lute) and the extent of leakage ( $\mathrm{p}$-value $=0.606, \mathrm{p}>0.05$ ). No leakage was observed in $46.6 \%$ of specimens using 


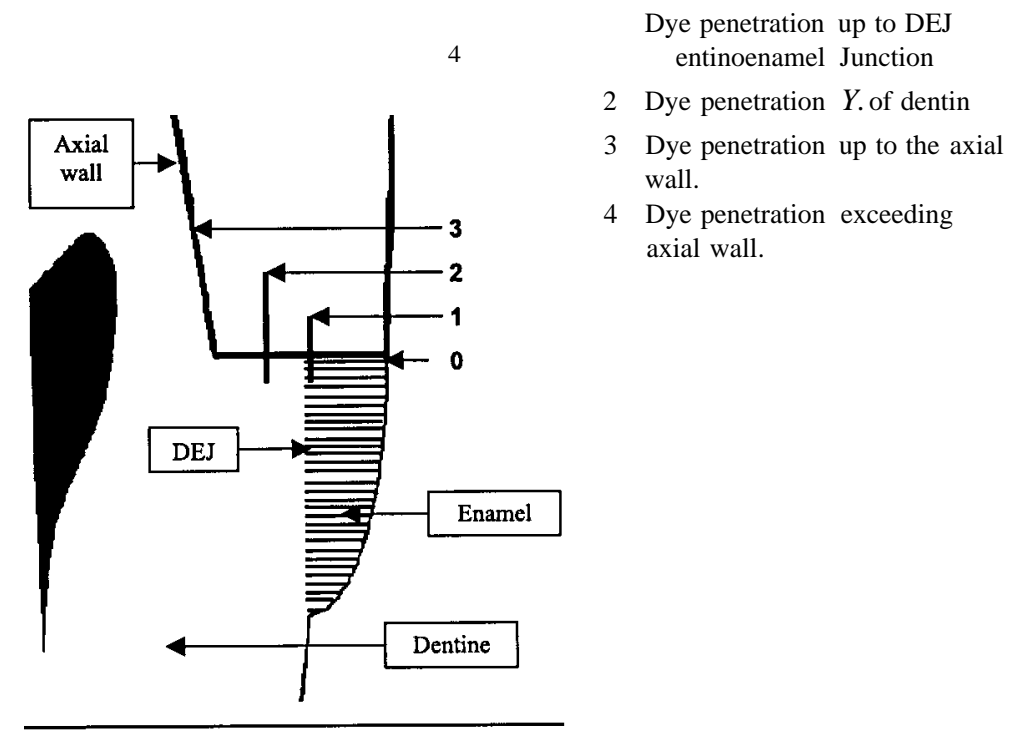

Figure 3: The site and degree of leakage of each section scoring system.

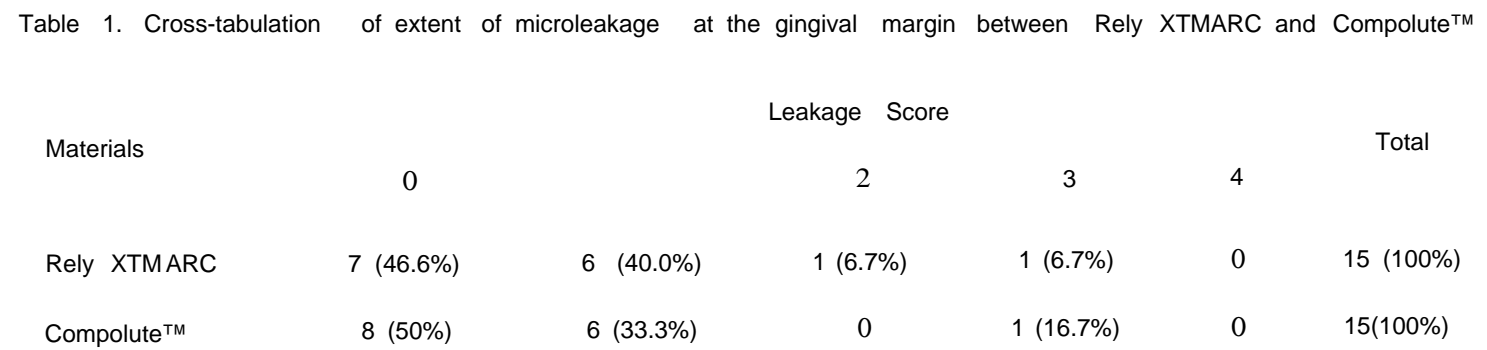

Rely $\mathrm{X}^{\mathrm{TM}} \mathrm{ARC}$ and $50.0 \%$ of specimens for Compolute'M(Table 1).

\section{DISCUSSION}

Consistency of the scoring was accomplished within the three evaluations of all the specimens. The interexaminer calibration was $89 \%$, giving a favourable indication of the consistency of evaluation. In this study, microleakage occurred in both groups (Rely XTM ARC and CompoluteT $>$ I)Most of the leakage occurred at the cement - tooth interface. The pattern of leakage in this study showed that with Rely XTMARC (Table 1), 7 specimens (46.6\%) Scored 0 (Figure 6a), 6 specimens (40.0\%) Scored I (Figure 6b), I specimen (6.7\%) Scored 2 and 1 specimen (6.7\%) Scored 3. It showed that most of the leakage occurs at the enamel interface before the dentoenamel junction. Only two specimens $(13.4 \%)$ showed leakage beyond the dentoenamel junction and none of the specimens had dye penetration beyond the axial wall. In Compolute ${ }^{\mathrm{TM}}$
0 No dye penetration

Dye penetration up to DEJ

Dye penetration $Y$. of dentin

Dye penetration up to the axial

Dye penetration exceeding axial wall 


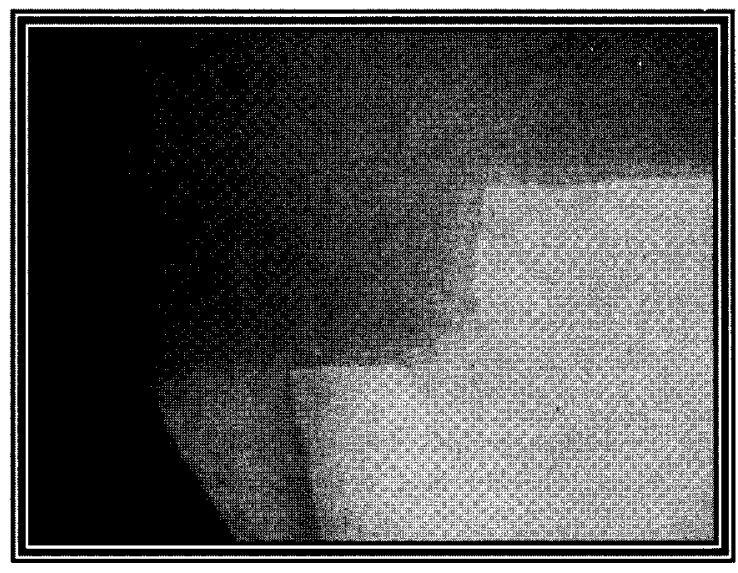

Figure 6a (Score 0)

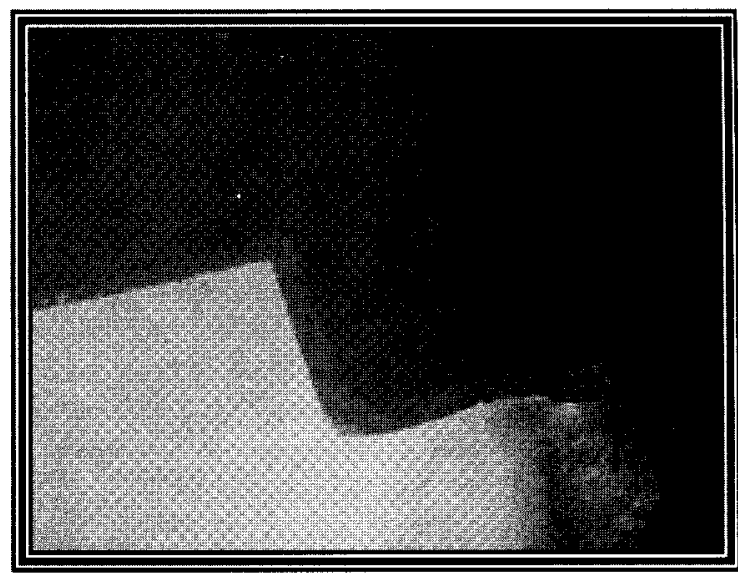

Figure $6 \mathrm{~b}($ Score I)

Figure 6a \& 6b: Rely""XARC, Score $=0$ and Score $=\mathrm{I}$.

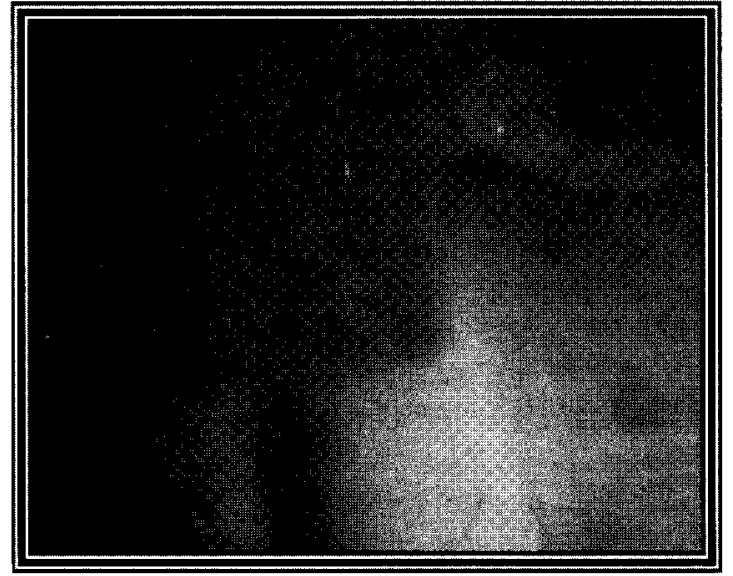

Figure 7a (Score 0)

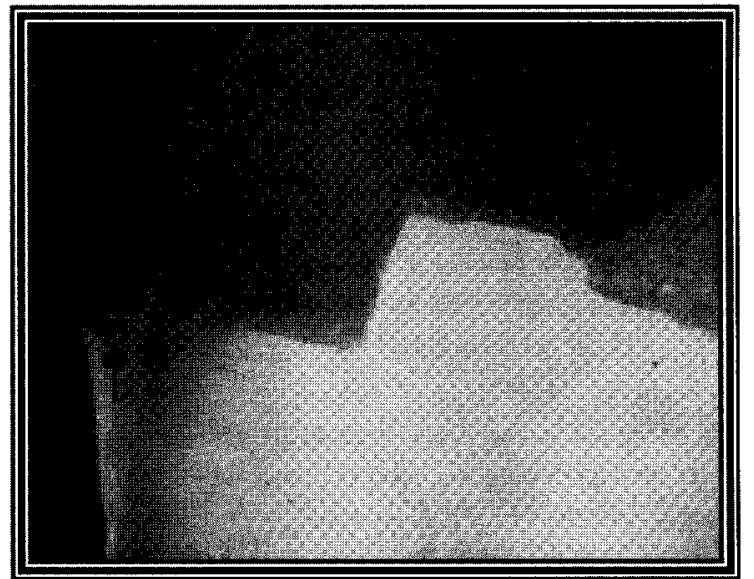

Figure $7 b($ Score 1)

Figure 7a \& 7b: Compolute ${ }^{\mathrm{TM}}$ Score $=0$ and Score $=1$.

bond at these two points, the stress applied may be sufficient to cause separation of the cement at either one or both of these interfaces. However in this study, none of the specimens showed leakage occurring at the cement-inlay interface. This observation is in accordance with the study by Alavi et al (10) who found that no leakage occurred at the cement - inlay interface. This could be due to chemical bonding between the composite and the resin luting cement or the surface was sandblasted to create a micromechanical retention (11) that prevented the separation between the inlay-cement layer and hence no microleakage happened at this interface.

The result of this study provides supporting evidence that the gingival margins are the weakest bonding area of indirect composite inlay. This is in agreement with the study done by Robinson et al (12), Hasegawa et al (13) and Shortall et al (11). However, compared to results obtained with the oldest systems, the superior sealing capacity of restorations which used these latest dentine bonding systems (EBS Multi and 3M ScotchbondT\$Single Adhesive) can be attributed to the hybridisation of the conditioned substrate (14).

In this present study, even though the scoring is in accordance to the scoring system used, the statistical analysis showed there was no significant difference between Rely XTMRC and Compolute ${ }^{\mathrm{TM}}(\mathrm{p}>0.05)$ in the extent of leakage. But this result should be interpreted carefully since the number of sample in this study was quite limited $(n=15)$. Therefore, it was suggested that two scores should be applied which either "No leakage" or "Leakage" (which include scores 1,2,3) (Table 2). Chi-Square test was done and it was found that there was no significant difference in the presence or absence of leakage, between Rely XTMRC and Compolute ${ }^{\mathrm{TM}}(\mathrm{p}>0.05)$.

The finding of this study is in agreement with Alavi et al (10) who found that there was no significant 


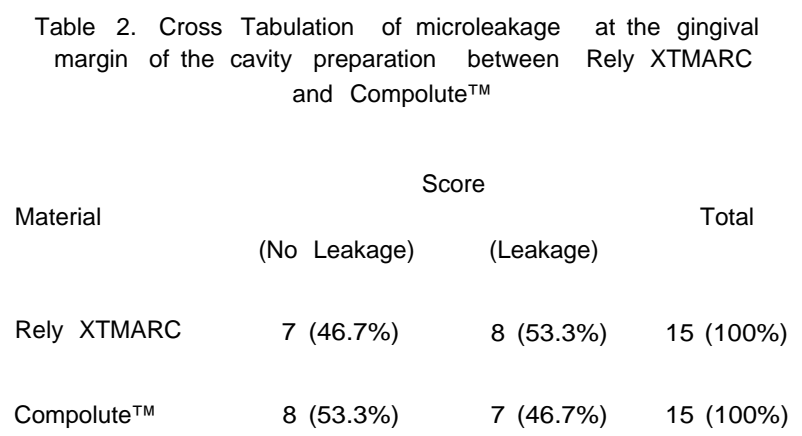

difference in the extent of leakage between several bonding systems and resin luting cements (including

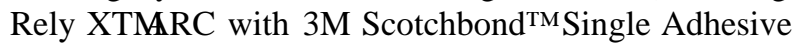
and Compolute ${ }^{\mathrm{TM}}$ with EBS Multi).

\section{CONCLUSIONS}

For both materials, leakage occurred most commonly within the enamel surface. There is no significant difference in the extent of leakage between Rely $\mathrm{X}^{\mathrm{TM}}$ ARC compared to Compolute ${ }^{\mathrm{TM}}$.In both cements (Rely XTMARC and CompoluteT leakage occurred between the cement-tooth interface. None of the specimens had leakage occurring between inlay - cement interfaces.

\section{REFERENCES}

1. Fayyad MA and Shorthall ACC. Microleakage of dentine bonded posterior composite restorations. J Dent 1987; 15: 67-72.

2. Peutzfeldt, A. Resin composites in dentistry: The monomer systems. Eur J Oral Sci 1997; 105(2): 97-116.

3. Shortall ACC and Baylis RL. Microleakage around direct composite inlays. J Dent 1991; 19: 307-11.

4. Ferracane JL. Using posterior composites appropriately. J Am Dent Assoc 1992; 123(1): 538.
5. Mannhart J, Kunzelmann KH and Chen HY. Mechanical properties and wear behavior of lightcured packable composite resins. Dent Mater 2000; 16: 33-40.

6. Irie M, Suzuki K. Current luting cements: marginal gap formation of composite inlay and their mechanical properties. Dent Mater. 2001 Jul; 17(4): 347-53.

7. El-Mowafy OM, Rubo MH and EI-Badrady WA. Hardening of new resin cements cure through a ceramic inlay. Oper Dent 1999; 24: 38-44.

8. Piemjai M, Miyasaka K, Iwasaki Y, Nakabayashi N. Comparison of microleakage of three acid-base luting cements versus one resin-bonded cement for Class V direct composite inlays. J Prosthet Dent 2002; 88(6): 598-603.

9. Ferrari M, Mannocci F, Mason PN and Kugel G. In vitro leakage of resin-bonded all-porcelain crowns. J Adhes Dent 1999; 1(3): 233-42.

10. Alavi AA and Kianimanesh N. Microleakage of direct and indirect composite restorations with three dentin bonding agents. Oper Dent 2002; 27(1): 19-24.

11. Shortall AC, Baylis RL and Baylis MA. Marginal seal comparison between resin-bonded Class II porcelain inlays, posterior composite restorations and direct composite inlays. Int J Prosth 1989; 2: 217-23.

12. Robinson PB, Moore BK and Schwartz ML. Comparison of microleakage in direct and Indirect composite resin restorations in vitro. Oper Dent 1987; 12: 113.

13. Hasegawa EA, Boyer DB and Chan DC. Microleakage of indirect composite inlays. Dent Mater 1989; 5: 388-91.

14. Bayne SC, Heymann HO, Wilder AD and Peutzfeldt A. Resin composites in dentistry: The monomer systems. Eur J Oral Sci 1997; 105(2): 97-116. 